\title{
RESISTENCIA AO FLUXO DE AR DAS VAGENS DE AMENDOIM COM DIFERENTES PERCENTUAIS DE IMPUREZAS
}

\author{
FIGUEIREDO NETO, Acacio Figueiredo ${ }^{1}$ \\ DANTAS, Bárbara França ${ }^{2}$ \\ SILVA, José Castro ${ }^{3}$ \\ OLIVIER, Nelson Cárdenas ${ }^{4}$ \\ SILVA, Maraisa Ferreira ${ }^{5}$
}

\begin{abstract}
RESUMO: Diante das necessidades de conhecimento prévio da perda de carga a ser vencida pelo conjunto motorventilador, em sistemas de aeração e secagem, decorrente da resistência apresentada pela massa porosa ao fluxo de ar, com este trabalho pretendeu-se estudar a resistência com diferentes percentuais de impurezas ao fluxo de ar, utilizando-se o produto com teor de umidade de $8 \%$ b.u. Para realização do experimento, utilizou-se um equipamento devidamente projetado. Foram medidas as perdas de carga na coluna do produto limpo e misturado com níveis de 2, 4 e $6 \%$ de impureza fina. Consideraram-se impurezas finas materiais como galhos, cascas e resíduos com tamanho menor que a das vagens, provenientes do campo juntamente com o produto. $\mathrm{O}$ ar foi insuflado na massa de amendoim por meio de um conjunto motor de $0,75 \mathrm{cv}$ e ventilador centrífugo, dotado de diafragma para a obtenção dos fluxos de ar, os quais variaram entre $0,3 \mathrm{e} 1,1 \mathrm{~m}^{3} \cdot \mathrm{s}^{-1} \cdot \mathrm{m}^{-2}$. Os modelos ajustados para os diferentes percentuais de impureza apresentaram um comportamento satisfatório, com coeficientes de determinação acima de 0,97 . Concluiuse que na medida em que diferentes percentuais de impurezas foram adicionados à massa do produto, para qualquer fluxo de ar, ocorreu um aumento na perda de carga.
\end{abstract}

Palavras-Chave: Perda de carga. Secagem. Vagem de amendoim.

\section{THE RESISTANCE TO THE FLOW OF AIR OF THE BEANS OF PEANUT WITH DIFFERENT PERCENTILE OF IMPURITY}

SUMMARY: Due to the necessity of previous knowledge of the load loss to be won by the group motor-fan, in aeration and drying systems, due to the resistance presented by the porous mass to the flow of air, this work intended to study the resistance of the peanut in bean with different impurity of percentages to the flow of air, using the product with tenor of humidity of $8 \%$ wet basis. The testes were performed of the experiment, equipment was used properly projected. The losses of peanut bean load were measured in mixed with levels of 2, 4 and $6 \%$ of fine impurity. They were considered impurity fine fragments as branches, peels and residues with smaller size than the one of the beans, coming of the field together with the product. The air was insufflated in the peanut mass through a group $0,75 \mathrm{cv}$ motor and centrifugal fan, endowed with diaphragm for the obtaining of the flows of air, which varied between 0,3 and $1,1 \mathrm{~m}^{3} \cdot \mathrm{s}^{-1} \cdot \mathrm{m}^{-2}$. The adjusted models for the different ones percentile of impurity they presented a satisfactory behavior, with determination coefficients above 0,97. It was ended that the measure in that different percentile of impurity they were added to the mass of the product, for any flow of air, it happened an increase in the load loss.

Keywords: Load loss. Drying and peanut bean

\section{INTRODUÇÃO}

O amendoim (Arachis hypogaea L.) é uma das principais oleaginosas no Brasil e no mundo, tendo como principais países produtores a Índia, a China, os EUA, o Sudão e o Senegal. Sendo $80 \%$ da produção

\footnotetext{
${ }^{1}$ Engenheiro Agrônomo. Pós-Graduação na Engenharia Agrícola. Prof. UNIVASF, curso de Engenharia Agrícola e Ambiental, com área de atuação na tecnologia de produtos agrícolas.

${ }^{2}$ Pesquisadora da Embrapa Semiárido na área de Tecnologia de Sementes. UNIVASF.

${ }^{3}$ Professor da UNIVASF, do departamento de Engenharia Mecânica, atuante na área de fenômeno de transporte

${ }^{4}$ Professor da UNIVASF, do departamento de Engenharia Mecânica, atuante na área de Materiais e Processos.

${ }^{5}$ Acadêmica do Curso de Engenharia Agrícola da UNIVASF.
} 
mundial oriunda dos países em desenvolvimento e aproximadamente $67 \%$ dos trópicos semi-áridos (FIGUEIREDO, 1992; GONÇALVES et al., 2004). Considerado um alimento importante devido aos seus altos conteúdos de proteína e de óleo. Este produto é praticamente comercializado na vagem, onde a obtenção da renda gerada por este comércio promove um ganho a mais aos seus produtores (FAO, 2002).

A indústria de alimentos necessita, cada vez mais, de produtos mais homogêneos e com menor percentual de impurezas, e para tanto, a engenharia vem melhorando os processos de beneficiamento através das informações das propriedades físicas que surgem para os novos projetos de máquinas e equipamentos, evitando assim, desperdícios dos produtos.

O conhecimento da resistência ao fluxo de ar, quando este atravessa uma massa de produtos agrícolas, é fundamentalmente importante em projetos de equipamentos destinados ao pré-processamento e armazenamento (AGULLO; MARENYA,2005). Quando o ar é forçado a atravessar uma camada de produto granular, a resistência ao fluxo, também denominada perda de carga, desenvolve-se como resultado da perda de energia por atrito e turbulência gerados no meio poroso (CORRÊA et al., 2001).

Para ser consumido, o amendoim deve ser beneficiado após a colheita. Nesse processo, composto resumidamente pelas operações de beneficiamento, secagem, separação de vagem-grão, classificação, embalagem e expedição, a secagem é considerada, segundo Giuliani (1982), uma das etapas mais críticas, pois a condução inadequada dessa fase acarreta graves danos ao produto.

O sucesso dos processos de secagem e aeração depende da uniformidade de distribuição do ar no interior da massa de grãos que, por sua vez, depende da resistência que o produto oferece á passagem do fluido (HAQUE et al., 1982; DEVILLA, et al., 2005).

A resistência ao fluxo de ar em sistemas de secagem e aeração depende de vários fatores. Esta resistência não depende apenas do fluxo de ar e da altura da camada de grãos, mas também da presença de impurezas, do tamanho e da distribuição dos grãos.

A perda de pressão do fluxo de ar através da massa de produtos agrícolas depende do percentual de impurezas, pois uma massa de grãos ou vagens contendo grande quantidade de pó e fragmentos do produto apresentam menos espaços ao deslocamento do fluxo de ar. $\mathrm{O}$ conhecimento deste fator e sua relação são essenciais aos cálculos dos processos de secagem, limpeza, classificação e armazenamento. A resistência ao escoamento de ar varia quando impurezas são adicionadas ao produto, ressaltando-se que esta resistência é inversamente proporcional ao tamanho das impurezas (SHEDD, 1951; GRAMA et al., 1984).

Sabe-se que para se obter um armazenamento eficiente de grãos agrícolas, a granel, o produto deve ser submetido a operações de secagem e de aeração. A execução desses processos prolonga o período de armazenamento e evita a deterioração do produto, resultante de sua atividade biológica.

Desta forma o presente trabalho teve por objetivos determinar os efeitos da densidade de fluxo de ar e da quantidade das impurezas finas sobre a perda de carga em uma massa granular de amendoim em vagem.

\section{MATERIAL E MÉTODOS}

Foram utilizadas vagens de amendoim da variedade Tatu vermelho, as mesmas foram colhidas no campo experimental do Colegiado de Engenharia Agrícola do Campus de Juazeiro - BA da UNIVASF (Universidade Federal do Vale do São Francisco) no mês de setembro de 2010 e foram secas até atingirem o teor de umidade de $8 \%$ b.u.

Os frutos de amendoim foram colocados no interior do protótipo, esquematizado na Figura 1, utilizando as mesmas dimensões do protótipo construído por AFONSO (1994), onde o ar foi insuflado na massa de vagens de amendoim, por meio de um conjunto com motor de $0,75 \mathrm{cv}$ e ventilador centrífugo dotado de diafragma para obtenção dos diferentes fluxos de ar de 0 a $1,1 \mathrm{~m}^{3} \cdot \mathrm{s}^{-1} \cdot \mathrm{m}^{-2}$. As medidas de pressão 
estática, em cada ponto ao longo da coluna de vagens, foram efetuadas, utilizando-se um manômetro de tubo inclinado.

Figura 1. Esquema do protótipo utilizado na determinação do gradiente de pressão estática.

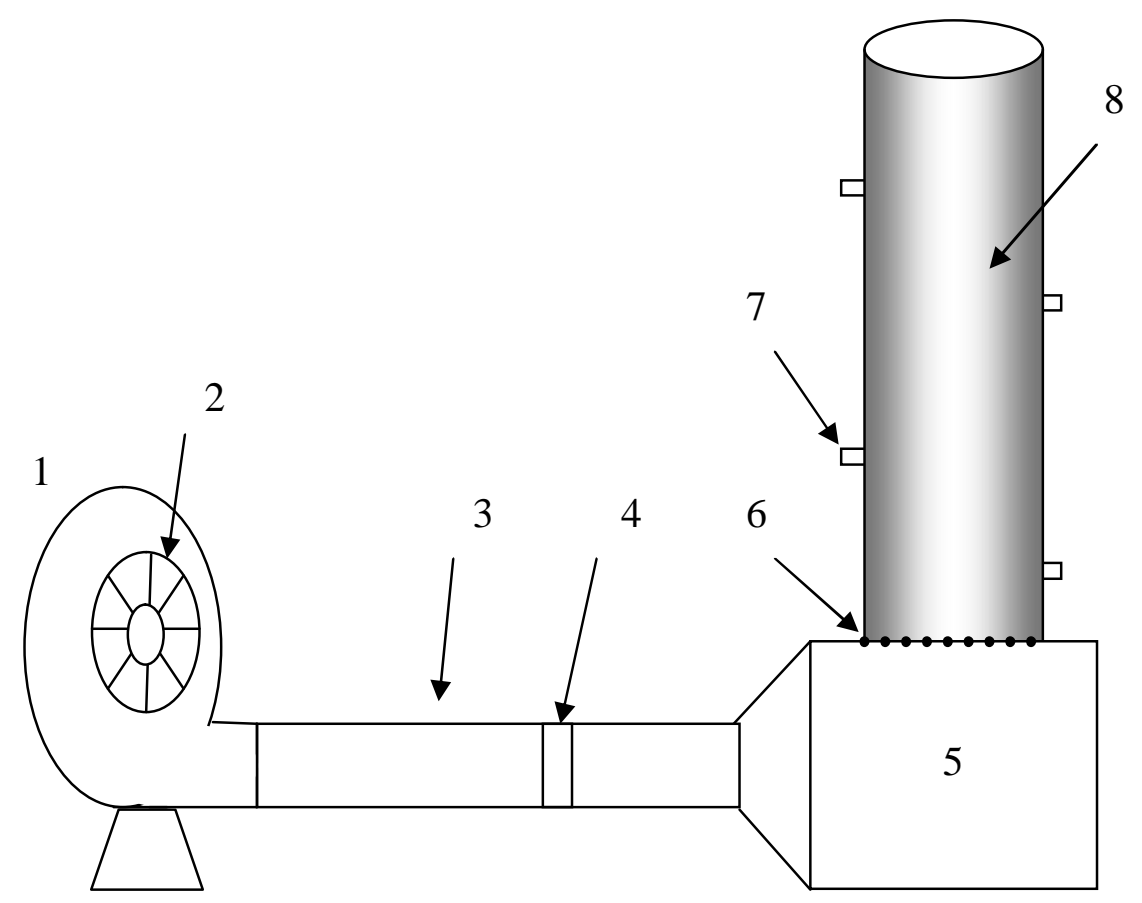

Em que:

1 - Ventilador do tipo centrífugo de pás retas - Motor de 0,75 CV;

2 - Diafragma para variação do fluxo de ar na coluna de grãos;

3 - Tubo de transição. Função: conduzir o ar insuflado pelo ventilador até o plênum;

4 - Homogeneizador do fluxo de ar;

5 - Plênum com função de homogeneizar a pressão;

6 - Piso perfurado feito com tela quadrada sobre uma chapa de aço perfurada fixa no fundo da coluna;

7 - Tomada de pressão estática;

8 - Coluna circular (chapa galvanizada) com altura de $1 \mathrm{~m}$ e diâmetro de $30,5 \mathrm{~cm}$.

As vagens de amendoim, limpos e manualmente selecionados, foram considerados com $0 \%$ de impurezas (Figura 2). Os demais percentuais foram obtidos com a adição de 2, 4 e $6 \%$ de impurezas, em peso, de material estranho em relação ao total da massa granular depositada na coluna. Foram consideradas impurezas, materiais como galhos, grãos de outras espécies, hastes, cascas, películas e rabiças que se encontravam destacadas das vagens, com tamanho menor que as das vagens de amendoim. A mistura das impurezas em cada percentual foi realizada em caixa plástica de modo a obter uma mistura homogênea de amendoim e impurezas. Estas impurezas utilizadas neste trabalho levam em consideração que os produtos agrícolas, quando submetidos a processos de secagem e, ou aeração, geralmente apresentam níveis de impureza de até 6\% (CORRÊA et al., 2001). 
Figura 2. Apresentação das impurezas e das vagens de amendoim secas e limpas, Juazeiro

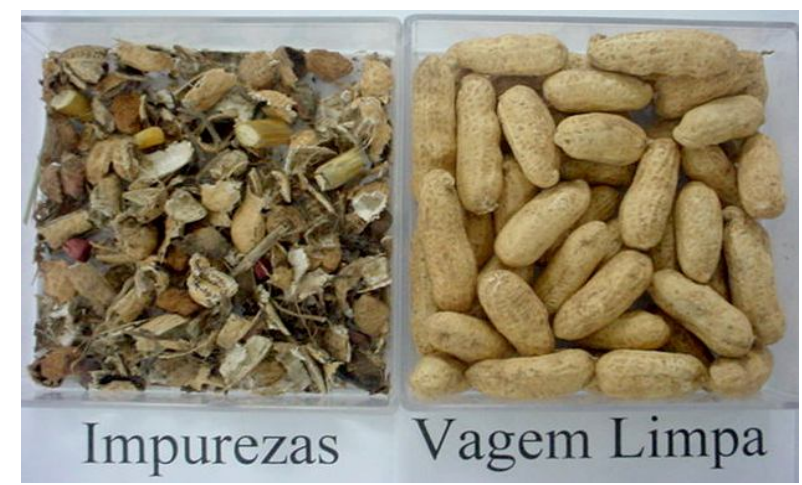

Fonte: Brasil, 2010.

Em cada teste, o enchimento da coluna com as vagens de amendoim foi feito com o produto em queda livre de um funil situado a $1 \mathrm{~m}$ de altura, a partir do fundo da coluna. Neste procedimento procurouse trabalhar com a mesma compactação do produto na coluna. As velocidades do ar foram obtidas com o auxílio de um anemômetro de fio quente, devidamente calibrado.

Os dados experimentais foram comparados com os valores calculados pelos modelos, analisandose o coeficiente de determinação da regressão e o erro médio estimado (SE) para cada modelo.

De acordo com Haque et al. (1978) e Guimarães (1998) estudando a perda de carga em uma camada de grãos, propuseram os seguintes modelos para estimar a perda de carga em função da densidade de fluxo de ar e do teor de impureza, respectivamente:

$$
\begin{aligned}
& \Delta P=a Q+b Q^{2}+c Q(I) \\
& \Delta P=a Q^{b}+I^{c}
\end{aligned}
$$

Em que:
$\Delta P=$
Perda de carga, Pa.m $\mathrm{m}^{-1}$
$Q=$ Fluxo de ar, $\mathrm{m}^{3} \cdot \mathrm{s}^{-1} \cdot \mathrm{m}^{2}$;
$a, b e c=$
Constantes dos modelos;
$I=$
Teor de impureza, decimal.

\section{RESULTADOS E DISCUSSÃO}

Na Tabela 1 pode-se observar que os dois modelos aplicados para os ajustes dos diferentes percentuais de impureza apresentaram um comportamento satisfatório, com coeficientes de determinação acima de 0,97. No entanto o modelo de Haque, além de apresentar o valor deste coeficiente superior a 0,98 , foi o que apresentou o menor valor de erro médio estimado. 
Tabela 1 - Resultados do ajuste aos modelos transformados de Haque e Guimarães para os dados obtidos com diferentes percentuais de impurezas.

\begin{tabular}{|c|c|c|c|c|}
\hline Modelos & Parâmetros & SE & $\mathbf{R}^{2}(\%)$ & Tendência \\
\hline \multirow{3}{*}{ Haque (1978) } & $\mathrm{a}=41,3362^{* *}$ & \multirow{3}{*}{11,16} & \multirow{3}{*}{98,29} & \multirow{3}{*}{ Aleatória } \\
\hline & $\mathrm{b}={ }_{*}^{144,7040^{*}}$ & & & \\
\hline & $c=8,4868^{* *}$ & & & \\
\hline $\begin{array}{l}\text { Guimarães } \\
\text { (1998) }\end{array}$ & $\begin{array}{l}\mathrm{a}=196,7458^{*} \\
\mathrm{~b}=1,8374^{* *} \\
\mathrm{c}=4,8816^{* *}\end{array}$ & 14,47 & 97,13 & Aleatório \\
\hline
\end{tabular}

Na Figura 3, apresenta-se a distribuição dos resíduos em função dos valores estimados para cada modelo ajustado de perda de carga com diferentes teores de impureza. Comprova-se que as distribuições de resíduos para os modelos de Haque e Guimarães apresentaram um comportamento aleatório indicando ser estes modelos apropriados para descrição dos ajustes matemáticos de perda de carga da vagem de amendoim.

Figura 3. Distribuição dos resíduos para cada modelo ajustado em função dos valores estimados de perda de carga com diferentes percentuais de impurezas da massa de amendoim.

Haque (1978)

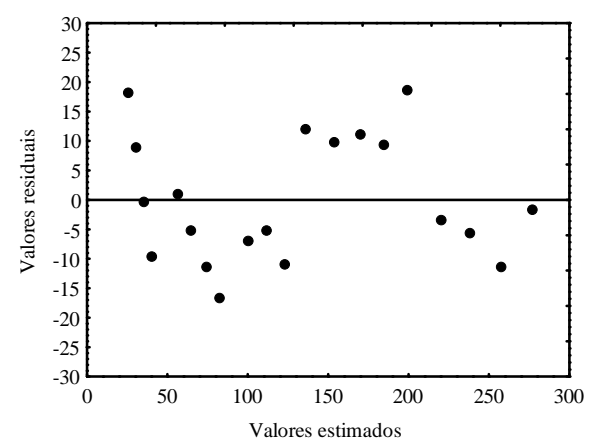

Guimarães (1998)

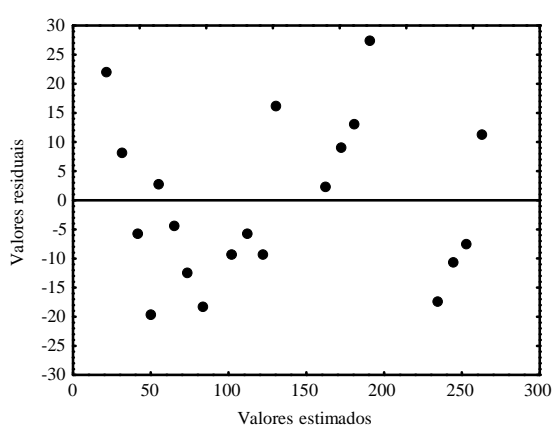

Na Figura 4, com a média dos dados observados de perda de carga observa-se que à medida que se aumenta o nível de impureza fina juntamente com o aumento da densidade de fluxo ocorre o aumento da resistência ao escoamento de ar. Resultados semelhantes foram obtidos por Jayas (1991).

Verifica-se também que a densidade de fluxo de ar para os percentuais de impurezas de 2 e $4 \%$ foram praticamente iguais inicialmente, ocorrendo um aumento de perda de carga quando atingem a densidade de $0,5 \mathrm{~m}^{3} \cdot \mathrm{s}^{-1} \cdot \mathrm{m}^{-2}$, concordando com as investigações realizadas por Grama et al.(1996). 
Figura 4. Perda de carga média observada através da massa de vagens de amendoim $\left(\mathrm{Pa}_{\mathrm{m}} \mathrm{m}^{-1}\right)$ em função da densidade de fluxo de $\operatorname{ar}\left(\mathrm{m}^{3} \cdot \mathrm{s}^{-1} \cdot \mathrm{m}^{-2}\right)$ e percentuais de impurezas.

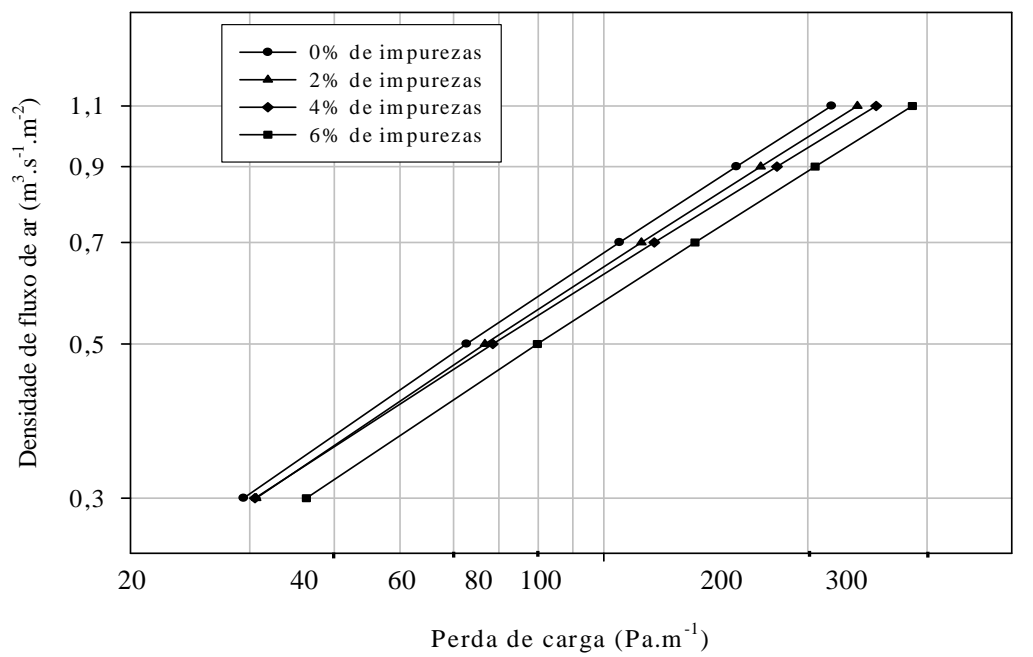

Na figura 5 são mostrados detalhes particulares de como a resistência do ar varia com a velocidade do ar para diferentes produtos limpos e secos. Nota-se, por exemplo, que a resistência oferecida por uma camada de soja á passagem do ar, numa velocidade específica, é de trinta vezes maior do que aquela oferecida para a mesma camada de vagem de amendoim. Consequentemente, se o mesmo ventilador e o mesmo motor forem usados para a secagem de ambos os produtos, considerando-se que a mesma velocidade do ar deverá passar pelas sementes, a camada de soja, que deverá ser colocada no secador, será próxima a um décimo da camada de vagem de amendoim.

Figura 5. Valores de perda de carga $\left(\mathrm{Pa}^{\mathrm{m}} \mathrm{m}^{-1}\right)$ em função do fluxo de $\operatorname{ar}\left(\mathrm{m}^{3} \cdot \mathrm{s}^{-1} \cdot \mathrm{m}^{-2}\right)$ para vagens de amendoim comparados com soja, milho e girassol

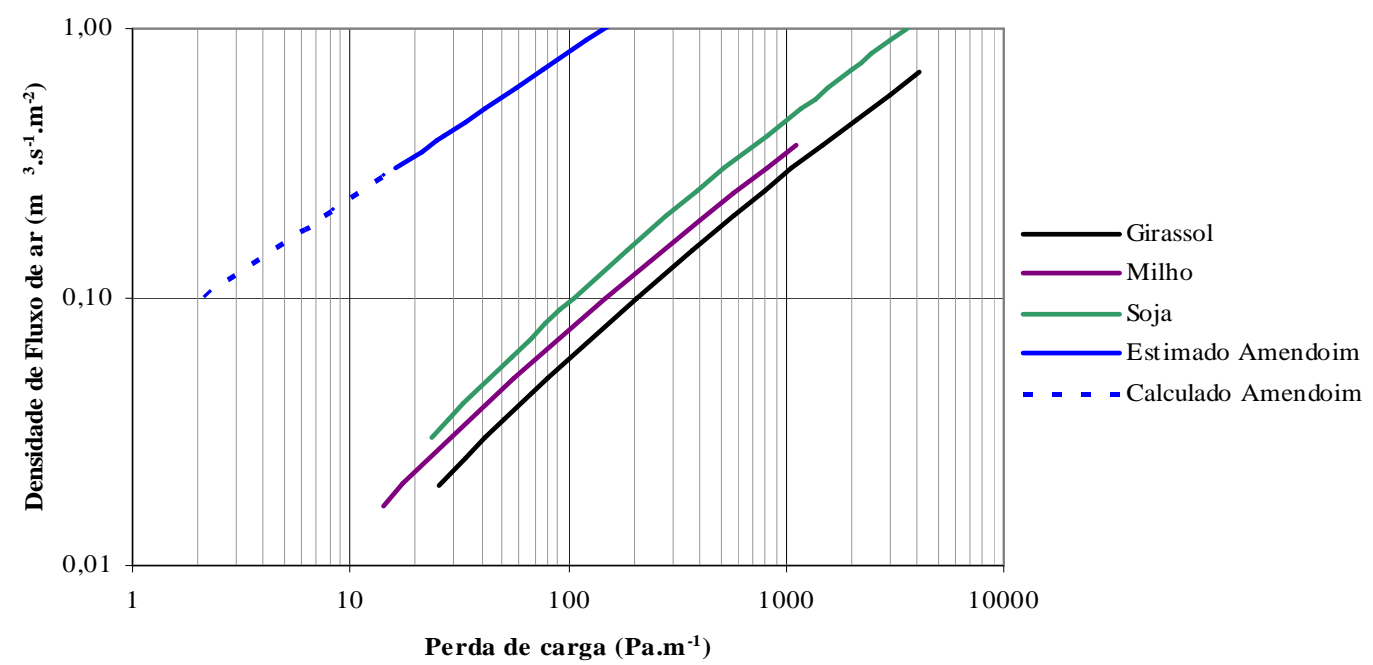

\section{CONCLUSÃO}

Conclui-se que na medida em que os diferentes percentuais de impurezas menores que as vagens foram adicionadas à massa do produto, para qualquer fluxo de ar, ocorreu um aumento na perda de carga das vagens de amendoim. 
Os modelos ajustados para os diferentes percentuais de impureza apresentaram um comportamento satisfatório, com coeficientes de determinação acima de 0,97 , sendo o modelo de Haque o que apresentou melhor os resultados. Com este modelo encontrado facilita a projeção de dimensionamento de equipamentos para secagem e aeração na indústria de pós-colheita de grãos.

\section{REFERÊNCIAS}

AFONSO, A.D.L. Gradiente de pressão estática em camadas de frutos de café (Coffea arábica $\mathbf{L}$.) com diferentes teores de umidade. 1994, 68p. Dissertação (Mestrado em Eng. Agrícola) Universidade Federal de Viçosa.

AGULLO, J.O., MARENYA, M.O. Airflow resistance of parchment arabica coffe. Biosystems Engineering, v.91, n.2, p.149-156. 2005.

CORRÊA, P.C., GUIMARÃES, W.T., ANDRADE, E.T. Resistência ao fluxo de ar em camadas de grãos de feijão afetada pelo teor de impureza. Revista Brasileira de Armazenamento. Viçosa, v.26, p.53-59, 2001.

DEVILLA, I.A., COUTO, S.M., QUEIROZ, D.M. Distribuição do fluxo de ar em silos com sistema de aeração: análise por elementos finitos. Revista Brasileira de Engenharia Agrícola e Ambiental. Campina Grande - PB, v.9, n.2, 2005.

FAO - Food and Agricultural Organization. Crop Production Statistics. Rome, Italy, 2002.

FIGUEIREDO, D.J.C. Comportamento e estabilidade fenotípica em genótipos de amendoim nos Estados da Paraíba e Pernambuco. 1992. 54p. Dissertação (Mestrado). Universidade Federal da Paraíba. Areia.

GONÇALVES, J.A., PEIXOTO, C.P., LEDO, C.A.S. Componentes de produção de amendoim em diferentes arranjos espaciais no Recôncavo Baiano. Revista Brasileira de Oleaginosas e Fibrosas, Campina Grande, v.8, n.2/3, p.801-812, 2004.

GUIMARÃES, W.T. Efeito do nível e do tipo de impurezas na resistência ao fluxo de ar em camadas de grãos de feijão. 1998. 47p. Dissertação (Mestrado). Universidade Federal de Viçosa.

GRAMA, S.N.; BERN, C.J.; HURBURGH Jr., C.R. Airflow resistance of mixtures of shelled corn and fines. Journal Agricutural Engineering Research, Silsoe, n.63, p.73-86, 1996.

GIULIANI, F. La macadamia. Rivista di Agricoltura Subtropicale e Tropicale, São Jose, Costa Rica, v.76, n.1/2, p.103-61, 1982.

HAQUE, E.; AHMED, Y.N.; DEYOE, C.W. Static pressure drop in a fixed bed of grains as affected by grain moisture content. Transactions of the ASAE, St. Joseph, v.25, n.4, p.1095-1098, 1982.

HAQUE, E.et al. Static pressure drop across a bed of corn and fines. Transactions of the ASAE. St. Joseph, v.21, n.5, p.997-1000, 1978.

JAYAS, D.S., ALGUSUNDARAM, D., IRVINE, D.A. Resistence to airflow through bulk flax seed as affected by moisture content, direction of airflaow and foreign material. Canadian Agricultural Engineering. Saskatoon, v.32, n.2, p.279-285, 1991.

SHEDD, C.K. Some new data on resistance of grains to airflow. Agricultural Engineering, St. Joseph, v.32, n.9, p.493-495, 1951. 
